Proceedings of the 2009 Winter Simulation Conference

M. D. Rossetti, R. R. Hill, B. Johansson, A. Dunkin, and R. G. Ingalls, eds.

\title{
A SIMULATION FRAMEWORK TO EVALUATE AIRPORT GATE ALLOCATION POLICIES UNDER EXTREME DELAY CONDITIONS
}

\author{
Konstantinos Kontoyiannakis \\ Eduardo Serrano \\ Kevin Tse \\ Marcial Lapp \\ Amy Cohn \\ Industrial \& Operations Engineering Department \\ 1205 Beal Avenue, University of Michigan \\ Ann Arbor, MI 48109-2117, USA
}

\begin{abstract}
Severe weather can lead to significant runway capacity reductions. Runway priority is typically given to inbound flights, thus fewer flights depart and fewer gates become available for arriving aircraft, leading to delays on the tarmac. We provide a simulation-based framework for evaluating gate allocation policies under reduced runway capacity. We first analyze a simple example, demonstrating the complexity of the problem and some key insights into different operating policies. Having shown that even simple scenarios can be difficult (if not impossible) to evaluate in closed form, we turn to simulation. We model the impact of reduced runway capacity on inbound and outbound flights by considering a major U.S. airport and its legacy carrier, focusing on the impact of delays on passengers. The contributions of this work are to highlight the challenges of accurately modeling the impact of runway capacity reductions and to present a simulation-based framework for evaluating operational policies.
\end{abstract}

\section{INTRODUCTION}

Weather delays are an inevitable and often high-cost aspect of air travel. There is much interest in developing new methods to alleviate the impact of weather delays. For example, as reported by the Massachusetts Institute of Technology, News Office (2001), "[a] weather forecasting system developed by the Massachusetts Institute of Technology's Lincoln Laboratory and tested at four New York-area airports saved airlines and passengers more than \$150 million last year by reducing delays by 49,000 hours." Nonetheless, weather continues to play a substantial role in travel delays, as evidenced most recently by a snow storm on February 9, 2009 in Houston, TX that caused delays of up to seven hours, as reported by the Houston Business Journal (2009) Severe weather conditions can reduce outbound flight capacity at an airport, in the worst case requiring runways to close. Some inbound traffic, destined for the troubled airport, can be delayed by the Federal Aviation Administration (FAA) at the origin airport through a ground hold, or redirected to a different airport if already en route, but some, non-diverted aircraft will still need to land. If the outbound flow has been reduced, there may not be enough gates available for all inbound flights, as the delayed outbound flights continue to occupy them. In such cases, there are a number of alternatives to deal with these incoming aircraft. For example:

- Delay the inbound flight on the tarmac and wait until an outbound flight pushes back and frees a gate once the delay ends.

- Load the outbound flight, push back from the gate (freeing it for the inbound aircraft), and incur an outbound delay, while waiting on the tarmac until a departure slot becomes available.

- Move an empty (i.e. not yet boarded) outbound aircraft to the tarmac, allow the inbound flight to use the gate to unload, then swap back the aircraft.

Each of these decisions features different amounts and types (in terminal, at gate, and on tarmac) of passenger delay. As a starting point to investigate this challenging and important problem, we focus on passenger discomfort and inconvenience (which we broadly refer to as unhappiness). This is not to suggest that airport- or carrier- accrued delay costs are not important; we simply use passenger unhappiness for the purpose of demonstration. We begin by modeling the most simple 


\section{Kontoyiannakis, Serrano, Tse, Lapp and Cohn}

of examples one gate, one outbound aircraft currently occupying this gate, and one inbound aircraft that has just landed and is awaiting the gate so that it can unload. Motivated by the complexity of even this simple case, we then develop a simulation-based framework for evaluating different operational policies under different airport conditions.

Within this research, we have made a number of assumptions to overcome limitations on the data that was available to us, including the functional properties of passenger unhappiness. As a result, we do not suggest that our results are directly applicable to the real-world problem. Instead, our goal is to demonstrate how this simulation-based framework can be used by others, in the context of real-world data and situational expertise, to analyze and compare different scenarios.

\section{LITERATURE REVIEW}

Simulation models are used frequently in literature to overcome complex situations that appear in systems such as the departure process of an aircraft. Furthermore, the development of simulation frameworks of complex systems such as airports has been explored with respect to various processes. For example, Yan, Shieh, and Chen (2002) provide a simulation framework to determine optimal gate assignments under possible delay. Their work seeks to minimize passenger travel distance.

Gilbo (1997) provides a queuing model to analyze and optimize airport traffic flow. While the results presented in this paper are directly taken from queuing theory, it still provides an analysis as to the balance of flow across an airport, especially during reduced capacity. Our work differs in that we do not consider inbound/outbound resource allocation, but rather assume they are fixed. In addition, Gilbo (1997) does not take gate allocation and operational aircraft swapping procedures into consideration as presented in our work. As a direction of future research, it may be of interest to combine our simulation model with an effective resource allocation model, such as the one presented in this paper.

In addition to providing a decision analysis framework, simulation has also been used to provide insights into airport scenarios under severe delay conditions. Carr et al. (2002) use simulation to analyze the Boston-Logan airport under severe flow restrictions. The difference from our work is that we specifically focus on gate availability and measure passenger unhappiness accordingly. Carr et. al. (2002) also use an outbound flow reduction, but analyze the outbound queue state as a function of delay.

\section{SINGLE-GATE MODEL}

In this section, we introduce a simplified version of the problem. Specifically, we focus on a single-gate airport, where an outbound flight is currently docked and waiting to depart. An inbound aircraft arrives, which requires that gate in order to disembark. As noted previously, several possible operational strategies are available, which are analyzed in detail in this section. Prior to any analysis however, an equation that allows us to calculate total passenger unhappiness, our objective, for each flight is required. We define our function parameters as follows:

$P \quad$ number of passengers on the flight

$t_{r} \quad$ delay time spent aboard aircraft parked on the tarmac

$t_{g} \quad$ delay time spent aboard aircraft parked at the gate

$t_{a} \quad$ delay time spent at the airport, i.e. inside the terminal

$\pi$ rate of unhappiness accumulation corresponding to the number of passengers aboard the flight

$r$ rate of unhappiness accumulation when the passenger incurs delay aboard an aircraft parked on the tarmac

$g$ rate of unhappiness accumulation when the passenger incurs delay aboard an aircraft parked at the gate

$a$ rate of unhappiness accumulation when the passenger incurs delay at the airport, i.e. inside the terminal

Incorporating all parameters mentioned above, we develop a function that measures "passenger unhappiness". This function is given in equation (1).

$$
P U\left(P, t_{r}, t_{g}, t_{a}\right)=(1+\pi)^{P}(1+r)^{t_{r}}(1+g)^{t_{g}}(1+a)^{t_{a}}
$$

We assume that passenger unhappiness increases exponentially with respect to delay time accumulated. We justify this fact with two observations. First, a ten minute delay aboard an inbound aircraft awaiting gate arrival is more uncomfortable when the passenger has been confined to the aircraft for a considerable period of time than when the passenger has not in-curred any delay beforehand. Second, all else being equal, it is preferable for ten passengers to get delayed by ten minutes each than for one passenger to experience 100 minutes of delay. Both observations can be incorporated mathematically by letting passenger unhappiness be an exponential function of time, forcing parameters $r, g$ and $a$ to be constrained between 0 and 1. We further assume that all passengers on the same flight incur the same amount of delay. We justify this assumption 


\section{Kontoyiannakis, Serrano, Tse, Lapp and Cohn}

by arguing that passengers are generally required to check-in 30-minutes prior to boarding. Transfer passengers, on the other hand, are given a 30-minute gate transfer time. These times force passengers to appear within a reasonably-close time window.

The equation also takes into account that passengers have different levels of unhappiness at different locations. Passengers are the least unhappy when they wait at the airport inside the terminal. The terminal has fewer restrictions and more sources of entertainment, as compared to the aircraft. Passengers are more unhappy when they are onboard, especially if parked on the tarmac. Passengers actions are confined on the aircraft and it is possible that the aircraft does not have sufficient resources, like food and water, to satisfy the needs of the passengers onboard when the delay becomes substantial. Therefore, this paper focuses on the situation where the rate of unhappiness accumulation is greatest when passengers incur delay on an aircraft parked on the tarmac, and the least when the passengers spend the same delay time in the terminal. Using our notation, we can represent this as $r>g>a$. Intuitively this strict inequality holds, and we demonstrate the relative size of each of the coefficients later in this section, as well as in our simulation section, section (4).

Intuition suggests that emotion contagion can take place in the cabin, where passengers can share and amplify unhappiness and frustration through speech and action. This network effect leads to a positive relationship between the number of passengers and the amount of unhappiness per passenger, similar to the relationship between delay incurred and passenger unhappiness. However, the sizes of these effects are different. Previously, we argued that the total unhappiness of ten passengers each suffering ten minutes of delay is better than one passenger undergoing 100 minutes of delay. This is reasonable even if the group of ten passengers is on the same aircraft. It follows that the exponential increase of passenger unhappiness due to a higher number of passengers is less than the exponential increase of unhappiness due to the increase in delay time. In other words, although passenger unhappiness is an exponential function of the number of passengers, $P$, the rate of unhappiness as related to the number of passengers on the aircraft, $\pi$, is relatively small.

Equation (1) is a basic equation for passenger unhappiness that addresses some of the most important variables. While other factors also affect passenger unhappiness, this equation is one example that illustrates how unhappiness can be expressed. It also provides us with a starting point from which to compare different ground delay-related policies and evaluate the complexity of the problem. We encourage further research to be done in developing a higher level approximation of passenger unhappiness in our Future Work section, especially one that can relate unhappiness to cost measured in monetary terms. With such an equation, one can weigh passengers costs against those suffered by the airport authorities and the airline companies when devising operating policies.

Using equation (1), we return to the example mentioned in the beginning of this section with only one gate but two aircraft one inbound and one outbound that require a gate. For this simple example, we assume that the outbound passengers are already onboard when the delay is announced and that the length of the delay is known. In this case, there are two choices to be made. First, which aircraft should occupy the gate? Second, should the outbound passengers wait out their delay on the aircraft or in the terminal? These two questions lead to four basic policies, which are shown in Table (1).

To calculate passenger unhappiness for each policy, we need to compute how long each passenger waits in the terminal, at the gate, and on the tarmac. In these calculations, only the time spent above and beyond the time needed for regular operations will cause accumulation of unhappiness. For example, the time spent by the inbound passengers to disembark does not generate unhappiness given that the passengers have to disembark regardless of delay. Taking these into account, the passenger unhappiness equations appear below as equations (2) through (5), using the following notation:

$\begin{array}{ll}A & \text { Inbound aircraft } \\ B & \text { Outbound aircraft } \\ P_{X} & \text { Number of passengers on aircraft } X \text { (where } X \text { is aircraft } A \text { or } B \text { ) } \\ t & \text { Time before outbound aircraft is allowed to depart } \\ u_{X} & \text { Time needed to unload aircraft } X \text { (where } X \text { is aircraft } A \text { or } B \text { ) } \\ l_{X} & \text { Time needed to load aircraft } X \text { (where } X \text { is aircraft } A \text { or } B \text { ) } \\ m & \text { Time required to physically move an aircraft from one location to another }\end{array}$

- Policy I ( $B$ waits at gate until allowed to depart, $A$ waits until gate is cleared):

$$
P U(t)=(1+\pi)^{P_{A}}(1+r)^{t}+(1+\pi)^{P_{B}}(1+g)^{t}
$$

- Policy II ( $B$ unloads and waits until $t-l_{B}$ to reload, $A$ sits on tarmac waiting):

$$
P U(t)=(1+\pi)^{P_{A}}(1+r)^{\max \left(t,\left(u_{B}+l_{B}\right)\right)}+(1+\pi)^{P_{B}}(1+g)^{u_{B}+l_{B}}(1+a)^{\left(t-u_{B}-l_{B}\right)^{+}}
$$




\section{Kontoyiannakis, Serrano, Tse, Lapp and Cohn}

- $\quad$ Policy III ( $B$ moves to tarmac, and waits until cleared to depart, $A$ takes available gate and unloads):

$$
P U(t)=(1+\pi)^{P_{B}}(1+r)^{t}
$$

- Policy IV ( $B$ unloads and leaves gate, $A$ moves to the gate and unloads its passengers and then moves out. $B$ then moves back in, waits until time $t-l_{B}$ to pick up its passengers and prepares for departure):

$$
P U(t)=(1+\pi)^{P_{A}}(1+r)^{t}+(1+\pi)^{P_{B}}(1+g)^{t}
$$

Table 1: Four Basic Policies for the Two Aircraft - One Gate Example

\begin{tabular}{|c|c|c|c|}
\hline & & \multicolumn{2}{|c|}{ Outbound Passengers } \\
\cline { 3 - 4 } & & Boarded at Gate & At Airport \\
\hline Aircraft Gate & Outbound & Policy I & Policy II \\
\cline { 2 - 4 } Priority & Inbound & Policy III & Policy IV \\
\hline
\end{tabular}

To show how these equations are derived, we examine Policy I in detail. The passenger unhappiness equation is reproduced here:

$$
P U(t)=(1+\pi)^{P_{A}}(1+r)^{t}+(1+\pi)^{P_{B}}(1+g)^{t}
$$

The first and second term represent the unhappiness per passenger for the inbound aircraft $A$ and the outbound aircraft $B$ respectively. Since $B$ waits at the gate until cleared to depart, $A$ waits until the only gate is open and spends the whole delay duration $(t)$ on the tarmac, while $B$ spends the same period of time at the gate. Hence, delay time spent waiting on the tarmac $\left(t_{r}\right)$ for the first term and delay time spent at the gate $\left(t_{g}\right)$ for the second term are both equal to $t$. The equations for the other three policies are developed similarly.

For Policy II, there can be two situations. Since the aircraft are required to perform a given set of actions, it may be that the time required for these actions exceeds the delay time. For example, outbound passengers are forced to disembark once the delay is announced. If the remaining delay after leaving the aircraft is shorter than the time it takes the passengers to re-turn to the plane, then the boarding takes place immediately. Therefore, the inbound flight $A$ has to wait on the tarmac for an amount of time equal to $u_{b}+l_{b}$ if $u_{b}+l_{b}>t$. It follows that passengers for $B$ spend $u_{b}+l_{b}$ amount of time getting off and back onto the aircraft, which has a rate of unhappiness accumulation of $g$ since these actions take place at the gate. They also spend $t-u_{b}-l_{b}$ at the airport if $t>u_{b}+l_{b}$.

In Policy III, $A$ incurs no delay since $B$ is forced swap from the gate immediately once the inbound flight requires a gate. Next $B$ waits on the tarmac for the whole duration of the delay.

Similar to Policy II, the actions that should be taken according to Policy IV may take a longer time to execute than the delay time itself. $A$ has to wait until $B$ finishes unloading before $A$ can move to the gate. Therefore $A$ incurs a delay of $u_{b}$ regardless of the length of the delay. For the outbound flight $B$, its passengers must disembark and wait in the terminal. The policy then requires the aircraft to swap positions, let $A$ unload and swap positions again. If the delay time less the time required to load and unload $B$ is greater than the time needed to execute these required actions, then the passengers of $B$ wait in the terminal until time $t-l_{b}$. Otherwise, $B$ 's passengers must wait until the required procedures are complete before they can return to their aircraft, even if the delay has ended. Here, we assumed that the swapping of the two aircraft can occur simultaneously, thus the time required to swap aircraft positions equals the time required to move a single aircraft.

To illustrate the operational policies above, we use Figure 1(a) and Figure 1(b) to show passenger unhappiness as a function of time. The parameters used for these particular graphs are shown in Table (2).

Table 2: Parameter Estimates

\begin{tabular}{|c|c|c|c|c|c|c|c|c|}
\hline$r$ & $g$ & $a$ & $\pi$ & $u_{a}$ & $u_{b}$ & $l_{a}$ & $l_{b}$ & $m$ \\
\hline 0.02 & 0.015 & 0.01 & 0.01 & 30 & 30 & 30 & 30 & 5 \\
\hline
\end{tabular}

Figures 1(a) and 1(b) show that Policy IV is the best policy to employ in this simple case, so long as the delay time $(t)$ is long. But there are drawbacks to Policy IV. Figure 1(a) illustrates that it is not the best policy when the delay $(t)$ is 


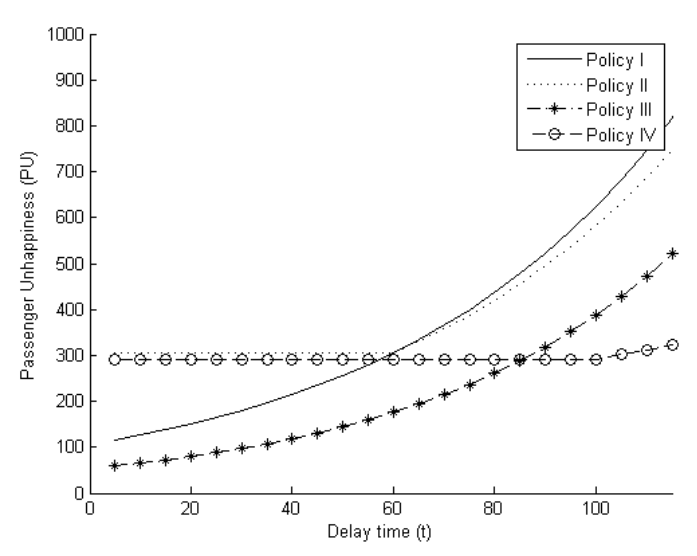

(a) Delay Time, $t<120 \mathrm{~min}$

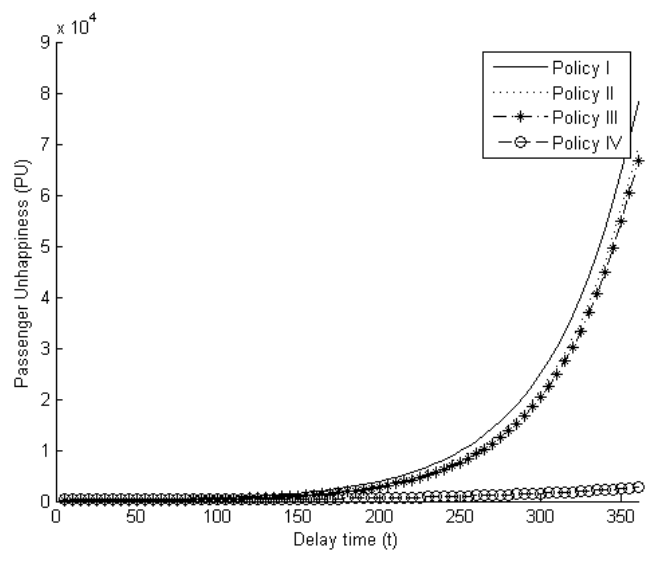

(b) Delay Time, $t<360 \mathrm{~min}$

Figure 1: Passenger Unhappiness for Different Policies

less than 85 minutes. Furthermore, Policy IV involves complex logistics. Policy IV requires the deboarding and reboarding of these passengers as well as multiple movements to perform the required aircraft swaps. These procedures in turn bring about additional costs and possible risks (movements of aircraft) to the carriers. Alternatively, Policy III, where the two aircraft swap positions immediately, produces less unhappiness than Policies I and II, and is also much simpler to execute than Policy IV.

This paper continues the analysis of passenger unhappiness based on the results found in this section. The focus will be on Policy III, whereby aircraft $B$ moves out onto the tarmac at a specific time, and aircraft $A$ is allowed to disembark. Although this is a viable policy, the next section of this paper investigates the effect of the change in swap time of the two aircraft on passenger unhappiness in an attempt to discover a better policy. The derivation of the optimal swap time, in turn, will then be incorporated into the simulation which follows in section (4).

\subsection{Time-predictive swapping}

In the previous section, we presented four possible policies to address a delay of known length. There are of course many other possible policies that could be employed. In this section, we continue to assume that the delay length is known, and focus on Policy III - moving aircraft $B$ away from the gate even though it is not yet cleared for departure, so as to make the gate available for aircraft $A$. However, we modify this policy to recognize that the swap does not need to happen right away - it can happen any time between the beginning of the time horizon and the end of the delay. Given the non-linearity of the unhappiness curve as a function of time, how can we choose the best swap time? In the analysis that follows, we show that under certain values of the passenger unhappiness parameters $(r, g, a)$, the optimal time to swap aircraft is between time zero and the end of the delay, time $(t)$. The equation for passenger unhappiness when swapping at time $(x)$ is shown as equation (7).

$$
P U(x)=(1+\pi)^{P_{A}}(1+r)^{x}+(1+\pi)^{P_{B}}(1+g)^{x}(1+r)^{t-x}
$$

In this scenario, the inbound aircraft $A$ and the outbound aircraft $B$ wait on the tarmac and at the gate respectively before the swap takes place. Therefore, aircraft $A$ incurs a delay of $x$ minutes on the tarmac, accumulating unhappiness at the rate of $r$. Similarly, $B$ spends $x$ minutes at the gate where unhappiness grows at the rate of $g$. After the swap, $A$ 's passengers disembark and incur no more delay. $B$, however, waits on the tarmac for the rest of the delay, accumulating unhappiness at the rate $r$ of for $t-x$ minutes before it can take off.

We note that the form of this equation is the sum of two exponential terms and is therefore a convex function. This al-lows us to find the optimal swap time by differentiating with respect to this swap-time $(x)$. The differentiated equation is given in equation (8).

$$
\frac{d P U(x)}{d x}=(1+\pi)^{P_{A}}(1+r)^{x} \ln (1+r)+(1+\pi)^{P_{B}}(1+g)^{x} \ln (1+g)(1+r)^{t-x}-(1+\pi)^{P_{B}}(1+g)^{x}(1+r)^{t-x} \ln (1+r)
$$




\section{Kontoyiannakis, Serrano, Tse, Lapp and Cohn}

Setting this equal to zero and solving for $x$ lets us determine the optimal swap time as seen in equation (9).

$$
-\left(\ln \left(-(1+\pi)^{P B-P A} \ln \left(\frac{1+g}{1+r}\right)(\ln (1+r))^{-1}\right)+\ln (1+r) t\right)(\ln (1+g)-2 \ln (1+r))^{-1}
$$

Next, we provide a numerical example to motivate the idea of an optimal swap time. Parameter assumptions are provided in Table (3).

Table 3: Parameter Estimates

\begin{tabular}{|c|c|c|c|c|c|}
\hline$P_{A}$ & $P_{B}$ & $r$ & $g$ & $a$ & $\pi$ \\
\hline 400 & 400 & 0.020 & 0.015 & 0.01 & 0.01 \\
\hline
\end{tabular}

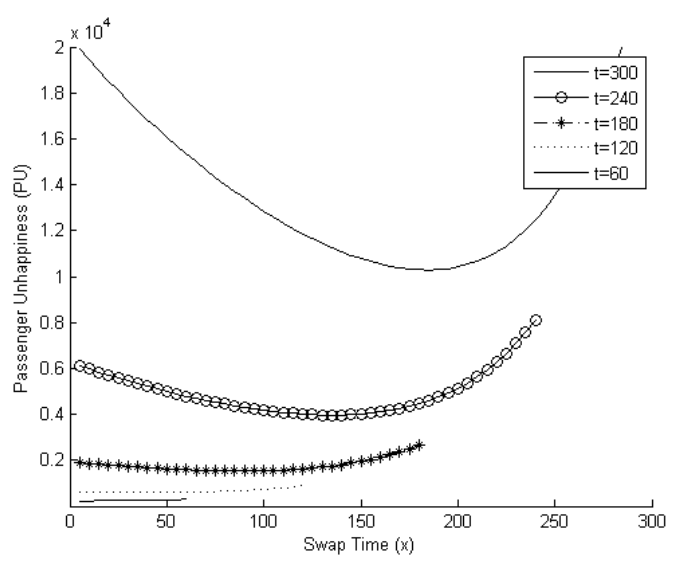

Figure 2: Passenger Unhappiness as a function of swap time

Figure (2) depicts various possible predictions for the total time of the delay. One should note from the graph that, the larger the predicted system delay $(t)$ is estimated to be, the more pronounced the swap time results.

Starting with equation (1) and its derivation to the development of several policies and the analysis of their respective unhappiness measures, as depicted by Figures 1(a) and 1(b) above, we have seen that even this very simple case is difficult to analyze in closed form. Using our measure of passenger unhappiness, we are able to find an optimal swap time to minimize this objective. A simulation framework is a valuable alternative that can provide us with a more realistic and efficient method of evaluating different policies. Another advantage to a simulation framework is that assumptions that we made for the closed form solution can be relaxed. For example, simulation can assess policies under the more realistic condition where the airport has multiple gates. Using the results derived in this section, we now turn to simulation to help model the more realistic, multi-gate airport scenario.

\section{MULTIPLE-GATE SIMULATION FRAMEWORK}

The example from section (3) provides an interesting framework to understand the intricacies of an airport system under delay, but also reveals its complexities and the difficulty of approaching the problem with a closed-form solution. As such, we turn to simulation to aid in analyzing the effects of different policies on passenger unhappiness. We use the Detroit/Wayne County Airport (DTW) as a basis for analysis, focusing on Terminal A of its flagship carrier, Northwest Airlines. This particular terminal provides a large enough terminal for our conclusions to be of interest, while allowing enough flexibility in the gate assignment policy during an extreme delay.

For the sake of exposition, we consider three policies and evaluate a separate simulation model for each. We assume that there exists a single delay that has completely closed the outbound runways for some stochastic period of time. Once this delay ends, the runways re-open and departures resume. The three policies considered are as follows: 


\section{Kontoyiannakis, Serrano, Tse, Lapp and Cohn}

1. All arriving aircraft proceed to an available gate. If all gates are full, inbound aircraft wait in an inbound queue until a gate is freed at the end of a delay.

2. All arriving aircraft proceed to an available gate. If all gates are occupied, the departing aircraft with the earliest scheduled departure time is moved away from the gate into an outbound queue and awaits departure on the tarmac.

3. All arriving aircraft proceed to an available gate. If all gates are occupied, the departing aircraft with the earliest scheduled departure time is moved away from the gate to await the remainder of the delay on the tarmac from an outbound queue, but only after an "optimal" swap time, as calculated in section (3.1), has passed.

The carrier data used to simulate the various policies described below was sourced from the Bureau of Transportation Statistics, using Northwest Airlines flights into and out of DTW on January 18, 2008.

<http://www.bts.gov/xml/ontimesummarystatistics/src/index.xml>

\subsection{Simulation Policies}

\subsubsection{Policy 1 - Inbound Aircraft Remain on Tarmac}

The first policy gives preference to departing passengers. Specifically, outbound aircraft will wait out the delay at their gate, while its passengers remain inside the terminal. Thirty minutes prior to scheduled departure, the aircraft will check for a de-lay. If there is no delay in the system, the aircraft will proceed to load its passengers. If a delay has occurred, the aircraft will wait to the end of the delay to perform the boarding procedure. If a delay event arrives during the loading of the aircraft, the aircraft will complete loading and subsequently wait at the gate with passengers aboard until the end of the delay. From section 3, we assumed that loaded passengers experience less unhappiness while sitting at the gate than on the tarmac.

Referring to Figure (4, but disregarding the shaded region, the aircraft will essentially wait at the gate for $x$ minutes, where in this policy $x$ is the remaining delay time, at the point of its scheduled departure.

In the meantime, arriving aircraft will begin to fill the remaining open gates, unloading their passengers, changing status to become an outbound flight, now preparing for their next departures. This can also be noted in Figure 4 after the "gate availability check" has been performed. If the delay is sufficiently long, all gates will eventually become filled. When this is the case, any additional arriving aircraft must remain in the inbound queue until the next aircraft reaches a condition where it can push back from the gate.

When the delay finally clears, departing aircraft with a scheduled departure time before the current time will get ready to leave in an earliest departure time fashion. If the aircraft began boarding before the start of the delay, the aircraft is already loaded and pushes back from its gate immediately; otherwise it must board all passengers, and thus incur a thirty minute wait before departing. As the departing aircraft release their gates, arriving aircraft are allowed to exit the inbound queue and unload at a free gate.

\subsubsection{Policy 2 - Commanding a Outbound Aircraft to Increase Gate Availability}

The following policy places emphasis on the inbound aircraft. More specifically, the system operates nominally until a delay event occurs, much like in previous policy. In this case, however, once all gates have been occupied by inbound aircraft, and a subsequent aircraft arrives, a "SEND" event is triggered. That is, the aircraft with the earliest scheduled departure time be-gins boarding its passengers to then move into the outbound queue. This situation can be summarized by stating: when an inbound aircraft cannot find a gate, an aircraft which was supposed to have departed already, but still remains at the gate due to the delay, is asked to board and subsequently depart from the gate to allow for the inbound aircraft to arrive. It could be the case that no aircraft is actually available for departure since a possible departure time has not been reached. In this case, the inbound aircraft remains in the inbound queue until an aircraft reaches its scheduled departure. This policy can also be seen visually in Figure (4), this time by including the shaded area, the "Send Departure Event" portion. In this policy, the waiting time $(x)$ in Figure (4) is essentially 0, forcing out a departing aircraft from the gate as soon as an inbound aircraft needs a gate to unload.

\subsubsection{Policy 3 - Commanding an Outbound Aircraft after Optimal Swap Time}

This last policy can most effectively be explained as a combination of the previous two policies. Again, on the onset of a delay, arriving aircraft begin to fill the remaining free gates. When gates reach saturation, a "SEND" command is sent to the departing aircraft with the earliest departure time. However, in this situation, the departing aircraft is allowed to stay at its 


\section{Kontoyiannakis, Serrano, Tse, Lapp and Cohn}

gate until a calculated swap-time given by equation (9) is reached. Referring to Figure (4), $x$ refers to the value of this swap time. This change, in essence allows the arriving and departing aircraft to split the total waiting time. Under the assumption that passenger unhappiness increases exponentially as a function of time, it makes logical sense that the splitting of wait time reduces the passenger unhappiness objective function. When the swap time is reached, the departing aircraft readies to move into the departure queue. If the aircraft began boarding prior to the start of the delay, the aircraft is already loaded and pushes back from its gate immediately and joins the outbound queue; otherwise it must load before leaving. Upon completing the boarding process, the arriving aircraft moves into the freed gate and unloads its passengers. The departing aircraft waits the remainder of the delay in the outbound queue.

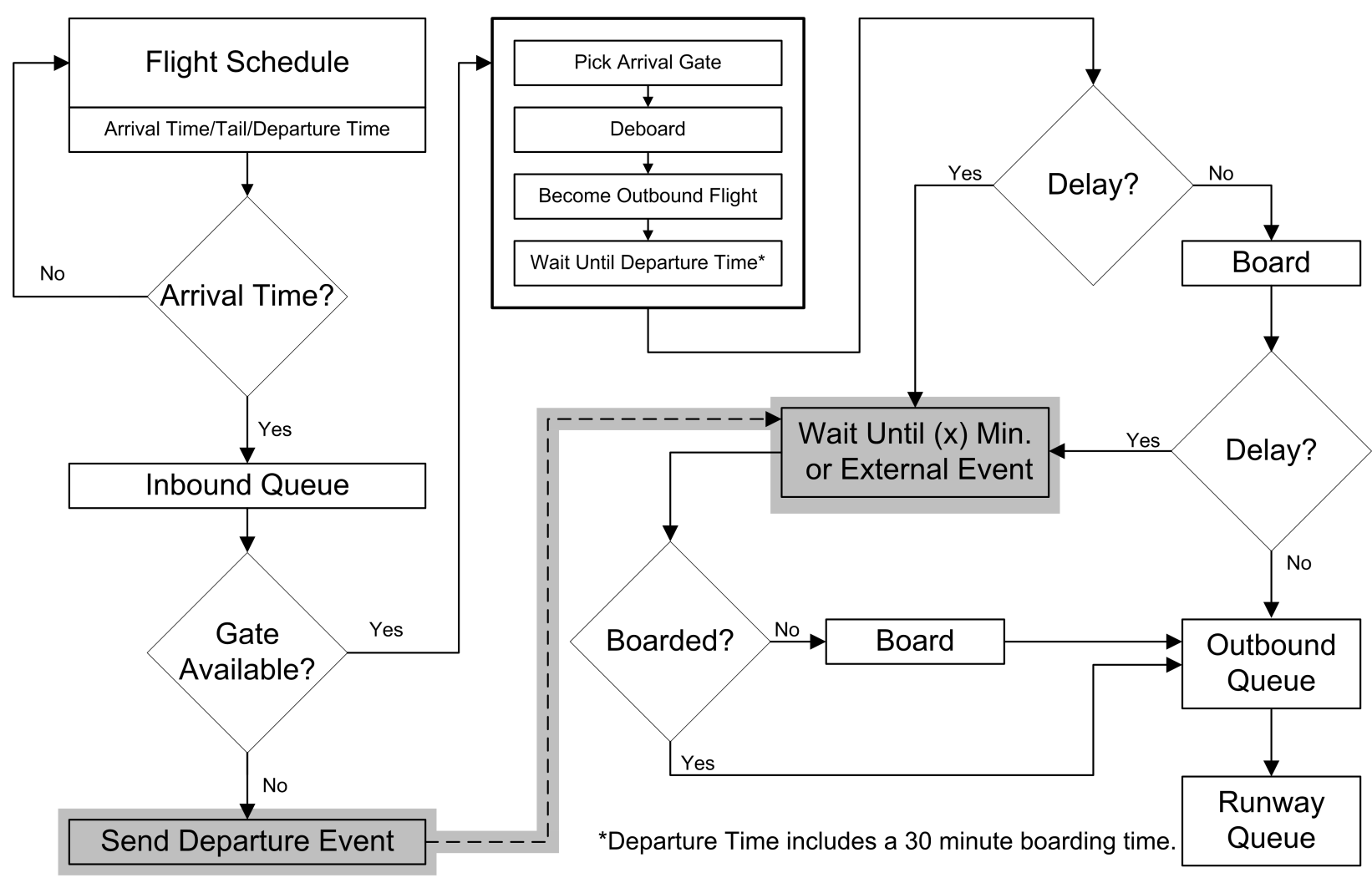

Figure 3: Simulation Framework Overview

\subsection{Numerical Example}

To show the effectiveness of the simulation framework and the policies described above, we provide a numerical example to help highlight the differences between alternate policies and their effect on the measure of passenger unhappiness. In this multiple policy simulation, we use the values presented in Table (2) as parameters for processing and calculating swap time. Aircraft arrivals are based on actual scheduled arrivals of Northwest Airline flights into DTW on January 18, 2008. We use a delay event which begins at 9 a.m., and lasts from 30 minutes up to a maximum of 4 hours. Under these conditions, our simulation models provide the results summarized in Table (4).

\subsubsection{Result Interpretation}

We use simulation to study the relationship between the inbound queue, gate availability and outbound queue. In addition, we assume that our delay time $(t)$ is a known value, such that each run of the simulation provides the total passenger unhappiness for a given value of $(t)$. 
Kontoyiannakis, Serrano, Tse, Lapp and Cohn

Table 4: Simulation Results based on Operations Policies 1, 2 and 3

\begin{tabular}{cccc} 
Delay Time $(\mathrm{min})$ & Policy 1 PU & Policy 2 PU & Policy 3 PU \\
\hline 30 & 2854.02 & 2853.59 & 2853.59 \\
60 & 2879.41 & 2875.97 & 2876.00 \\
90 & 2921.85 & 2912.59 & 2912.66 \\
120 & 2993.37 & 2969.97 & 2969.34 \\
150 & 3132.73 & 3022.39 & 3012.63 \\
180 & 3374.18 & 3125.90 & 3083.51 \\
210 & 3776.97 & 3307.45 & 3241.12 \\
240 & 4434.12 & 3634.04 & 3493.67 \\
\hline
\end{tabular}

From Table (4), we note the shaded values that represent the best policy for the given delay time value. Interestingly, for short delays, all policies perform extremely similarly. This is to be expected since the 30 minute boarding time accounts for much of the time that passengers spend waiting aboard the inbound aircraft on the tarmac. In addition, the gates do not necessarily have time to fill before departures can resume.

On the other hand, we can see that Policy 3, where a swap time is determined using the equation given in (9), outperforms the other two policies on longer delays. As the overall expected length of the delay increases, we see the pressure to get the inbound aircraft to a gate increase as well. This means that we do want to find a particular swap time at which to perform the aircraft swap. Furthermore, the simulation results presented in Table 4 are in agreement with our single-gate, numerical derivation in section 3.1 .

\subsection{Model Verification/Validation}

In order to verify and validate the accuracy of our simulation model, we turn to Sargent (2005). Verification consists of two steps: data verification and model verification. Our data, sourced from a major U.S. carrier, was verified to ensure that each inbound flight had a matching outbound flight, unless the aircraft remained on ground overnight. Furthermore, the simulation model was verified through black-box testing, ensuring each component functions individually. Finally, the simulation model itself was subjected to a variety of predefined test datasets to ensure it produced the expected output for a set of known parameter values.

Our validation process consisted of several measures. Our simulation is implemented in ProModel 7, a visual simulation programming tool. The software's sophisticated graphical features provided animation validation, ensuring the correct aircraft were going through the arrival, unloading/loading and subsequent departure process. Furthermore, we applied the operational graphics technique to understand our system during execution. Analyzing the queue sizes during nominal and delayed operations provided necessary insights into the correctness of the model.

\section{CONCLUSION AND FUTURE WORK}

Lengthy delay events, such as those attributed to severe weather conditions, can greatly impact airport operations. Operational decisions have to be made to manage arriving aircraft as gate availability decreases. Our simulation framework provides a proof-of-concept to evaluate a set of operational policies when managing an airport under delay conditions. From our preliminary analysis, using simple data assumptions, we hypothesize that using a policy that forces inbound aircraft to strictly wait on the tarmac (Policy 1) until a gate becomes available will be sub-optimal. More specifically, as inbound aircraft are required to wait on the tarmac, the passengers' unhappiness measure quickly exceeds that of those waiting at the gate.

We find that using a swap-time policy appears to perform better. In this operational policy, once an aircraft arrives and airport gates are unavailable, we determine the optimal swap time based on the balance between the passenger unhappiness that is incurred by both the inbound and the outbound aircraft. Once the swap time is reached, the outbound aircraft is forced to depart and wait on the tarmac, which allows the inbound aircraft to dock and debark its passengers. This result, while intuitive, is usually not applied in practice. Our results advocate a re-evaluation of current policies under severe delay conditions.

Furthermore, the complexity of this problem leads to several extensions of possible future research. First, our framework is built upon a passenger unhappiness measure, an exponential function that is based on intuition and experience. Further development of this function is warranted. In addition, when evaluating the trade-offs for different policies, one must also 


\section{Kontoyiannakis, Serrano, Tse, Lapp and Cohn}

consider direct and indirect costs incurred by the airlines. Although costs for measuring passenger unhappiness, aircraft swapping and other factors used in our framework will be non-trivial to quantify, the model itself can be easily extended to include new parameters and cost factors.

Another extension presents itself when assigning aircraft to gates. In our model, we assume that the inbound aircraft are free to dock at any available gate. This however, may not be a true representation, as airlines aim to minimize transfer distance between gates. This is done not only to minimize passenger travel distance between gates, but also luggage and crew transfer time. Incorporating this information in the gate decision process may pose additional trade-offs.

Finally, current simulation results are based on known delay times. While some information may be available at the time a system delay is incurred, exact delay values may not be known. An extension of the current simulation, based on historical data, could incorporate airport-based delay distributions for their respective delay values $(t)$.

Analyzing and simulating airports under severe weather conditions requires sophisticated models. Analytical solutions are difficult to evaluate beyond a single-gate scenario. Using a simulation-based framework, we can analyze and compare a set of operational policies and provide corresponding recommendations.

\section{ACKNOWLEDGMENTS}

We would like to acknowledge various industry experts who provided us with feedback and additional thoughts during the modeling and simulation process. This list is not limited to, but includes: Dr. John-Paul Clarke from Georgia Institute of Technology, Dr. Thomas Vossen from University of Colorado at Boulder, Dr. Bill Brunger from Continental Airlines, and Dr. Tim Jacobs from American Airlines.

\section{REFERENCES}

Carr, F., A. Evans, J.-P. Clarke, and E. Feron. 2002. Modeling and control of airport queueing dynamics under severe flow restrictions. In American Control Conference, 2002. Proceedings of the 2002, Volume 2, 1314-1319 vol.2.

Gilbo, E. P. 1997. Optimizing airport capacity utilization in air traffic flow management subject to constraints at arrival and departure fixes. IEEE Transactions on Control Systems Technology 5 (5): 490-503.

Houston Business Journal February 9, 2009. Bad weather hikes continental, expressjet delays. Houston Business Journal.

Massachusetts Institute of Technology, News Office February 15, 2001. Mit researchers save airlines and passengers $\$ 150 \mathrm{~m}$, reduce weather delays at new york airports. Massachusetts Institute of Technology, News Office.

Sargent, R. G. 2005. Verification and validation of simulation models. In Proceedings of the 2005 Winter Simulation Conference, ed. M. E. Kuhl, N. M. Steiger, F. B. Armstrong, and J. A. Joines, 130-143. Piscataway, New Jersey: Institute of Electrical and Electronics Engineers, Inc.

Yan, S., C.-Y. Shieh, and M. Chen. 2002. A simulation framework for evaluating airport gate assignments. Transportation Research Part A: Policy and Practice 36 (10): 885 - 898.

\section{AUTHOR BIOGRAPHIES}

KONSTANTINOS KONTOYIANNAKIS is an undergraduate student from Greece in the Industrial and Operations Engineering Department at the University of Michigan. His primary research interests lie in optimization modeling and statistical quality control analysis. His email is <kkontoyi@umich.edu>.

EDUARDO SERRANO is an undergraduate student in the Industrial and Operations Department at the University of Michigan. He is originally from Mexico and his research interests include operations optimization and decision analysis. His email is <guayosreumich.edu>.

KEVIN TSE is an undergraduate student from Hong Kong in the Industrial and Operations Engineering Department at the University of Michigan. His research interests lie in operations optimization and finance. His email is $\langle$ t sekev@umich. edu>.

MARCIAL LAPP is a graduate student in the Industrial and Operations Engineering Department at the University of Michigan. His research interests lie in modeling and solving large-scale optimization problems focused on the transportation and logistics industries. He holds a Masters and a Bachelors degree in Computer Science from the University of Michigan. His email is <mlappeumich.edu>. 


\section{Kontoyiannakis, Serrano, Tse, Lapp and Cohn}

AMY COHN is an Assistant Professor in the Industrial and Operations Engineering Department at the University of Michigan. She holds the Ph.D. in Operations Research from MIT. Her primary research interest is in applied discrete optimization problems, particularly in the airline industry. Her email address is <amycohn@umich.edu>. 